\title{
SEMISIMPLE MAXIMAL QUOTIENT RINGS
}

\author{
BY \\ FRANCIS L. SANDOMIERSKI $\left({ }^{1}\right)$
}

Notation and Introduction. $R$ denotes an arbitrary associative ring. A right $R$-module $A$ over $R$ will be denoted $A_{R}$. $B_{R}$ is a large submodule of $A_{R}\left(A_{R}\right.$ is an essential extension of $B_{R}$ ), if $B_{R}$ is a submodule of $A_{R}$ having nonzero intersection with every nonzero submodule of $A_{R}$. A right ideal $I$ of $R$ is a large right ideal, if $I_{R}$ is a large submodule of $R_{R}$.

Given $A_{R}, Z\left(A_{R}\right)$ is the singular submodule of $A_{R}$ [9], which consists of all those elements of $A$ whose annihilators in $R$ are large right ideals.

Following Johnson [9], $Q$ is a right quotient ring of $R$ if $Q$ is a ring with identity containing $R$ as a subring (the identity of $Q$ is the identity of $R$ if $R$ has one) and $R_{R}$ is a large submodule of $Q_{R}$.

The quotient rings considered by Goldie in [6], [7] will be called classical quotient rings. $Q$ is a classical right quotient of $R$ if every regular element (nonzero divisor) of $R$ is a unit in $Q$ and every element of $Q$ is of the form $a b^{-1}, a, b \in R, b$ regular in $R$. In general, a ring $R$ need not possess a classical right quotient ring.

Goldie [7], has given necessary and sufficient conditions that a ring possess a classical right quotient ring which is semisimple. Here semisimple means semisimple with minimum condition [8].

This paper is concerned with the question of characterizing those rings which have a semisimple maximal right quotient ring [4], [9], [10], [11] and in this case generalizing some simple well-known results about commutative integral domains, their quotient rings and modules over these domains. Johnson [9] has shown that $R$ has a regular maximal right quotient ring $Q$ if and only if $Z\left(R_{R}\right)=0$, where $Q$ is a regular ring [13] if every finitely generated right (left) ideal of $Q$ is generated by an idempotent. In this case $Q_{R}$ is injective [3] as a right $R$-module, hence the injective hull of $R$ [2].

A ring $R$ has a semisimple maximal right quotient ring $Q$ if and only if $Z\left(R_{R}\right)=0$ and $\operatorname{dim} R_{R}$ is finite, where a right $R$-module $M$ is of finite dimension if every direct sum of submodules of $M$ has only finitely many nonzero summands. This is the main result of $\S 1$. In addition another characterization is given for rings which possess a semisimple classical right quotient ring, namely, $R$ has a semisimple classical right quotient ring if and only if $Z\left(R_{R}\right)=0$ and if $I$ is a large right ideal of $R$, then there is an element $a \in I$ such that $a R$ is a large right ideal of $R$.

If $R$ has a semisimple classical right quotient ring $Q$, then it is known [3], that

Received by the editors February 10, 1966.

(1) Supported in part by National Science Foundation Grant GP-3993. 
$Q$ is the maximal right quotient ring of $R$. The converse is not valid, since there are rings with or without identity that have a semisimple maximal right quotient ring $Q$ and $Q$ is not the classical right quotient ring of $R$. Let $Q$ be the ring of $n \times n$ matrices over a division ring $\Delta$ and $R$ the set of upper triangular (strictly upper triangular) matrices of $Q$. It is easily verified that $Q$ is a right quotient ring of $R$ but $Q$ is not a classical right quotient ring of $R$. Since $Q$ is semisimple $Q$ is the maximal right quotient ring of $R$.

It is also shown in $\S 1$ that if $R$ has a semisimple maximal right quotient ring $Q$, then $Z(A / Z(A))=0$ for every right $R$-module $A$. This generalizes the analogous result if $R$ is a commutative integral domain, since then $Z\left(A_{R}\right)$ is the torsion subgroup of $A$.

In $\S 2$ rings with identity are considered.

In this case the following generalizations of results known [1] when $R$ is a commutative integral domain hold, thus extending some of the results of Gentile [5] also.

1. $R$ has a semisimple maximal right quotient ring $Q$ if and only if $A \rightarrow A \otimes_{R} Q$ has $Z\left(A_{R}\right)$ for its kernel for every unitary right $R$-module $A$.

If $R$ has a semisimple maximal right quotient ring $Q$, then

2. ${ }_{R} Q$ is flat [1] as a left $R$-module.

3. Every unitary left $Q$-module is flat as a left $R$-module.

4. If $Z\left(A_{R}\right)=0$, then $0 \rightarrow A \rightarrow A \otimes_{R} Q$ is the injective hull of $A$, a unitary $R$-module.

5. $\operatorname{Tor}_{1}^{R}(A, Q / R) \cong Z\left(A_{R}\right)$ for every unitary right $R$-module $A$.

Another result with weaker hypothesis is valid.

Any direct sum of injective right $R$-modules, each with zero singular submodule is injective if $\operatorname{dim} R_{R}$ is finite.

The following generalizes a result of Matlis [12]. If $H_{R}$ is an epimorphic image of an injective right $R$-module $E_{R}$ and $Z(H / Z(H))=0$, then $\left.Z(H)\right)$ is a direct summand of $H$ with complementary summand injective. The proof given here is simpler in that it does not appeal to any quotient ring of $R$ as was done in Matlis [12, Theorem 1.1] when $R$ is a commutative integral domain. Also Proposition 2.1, Proposition 2.2, and Proposition 2.4 of [12] can be generalized to a noncommutative ring $R$ which has a semisimple maximal right quotient ring utilizing identical proofs.

\section{Arbitrary rings.}

Definition 1.1. If $M$ is a right $R$-module, then the set of all large submodules of $M$ is denoted by $L\left(M_{R}\right)$.

It is useful to recall the following results, which are essentially in [9].

\section{Proposition 1.2.}

1. If $A, B \in L\left(M_{R}\right)$, then $A+B \in L\left(M_{R}\right)$ and $A \cap B \in L\left(M_{R}\right)$.

2. If $A \in L\left(M_{R}\right)$ and $B$ a submodule of $M$ containing $A$, then $B \in L\left(M_{R}\right)$. 
3. If $A$ is a submodule of $M$, then there exists a submodule $B$ of $M$ maximal with respect to the property that $A \cap B=0$ and consequently $A+B \in L\left(M_{R}\right)$.

4. If $f \in \operatorname{Hom}_{R}(M, N)$ and $A \in L\left(N_{R}\right)$, then $f^{-1}(A)=\{x \in M \mid f(x) \in A\} \in L\left(M_{R}\right)$.

Corollary. If $A_{1}, \ldots, A_{n} \in L\left(A_{R}\right)$ and $x_{1}, \ldots, x_{n} \in A$, then $I=\left\{r \in R \mid x_{i} r \in A_{i}\right.$ for all $i\} \in L\left(R_{R}\right)$.

Proof. $\left\{r \in R \mid x_{i} r \in A_{i}\right\}=I_{i}$ for each $i$ is the counter-image of $A_{i}$ by the right $R$ homomorphism from $R$ into $A$ given by left multiplication by $x_{i}$, hence $I$ is the intersection of finitely many large right ideals of $R$, so $I \in L\left(R_{R}\right)$.

Goldie [7] calls a right $R$-module $M$ of finite dimension, $\operatorname{dim} M_{R}$ finite, if every direct sum of nonzero submodules of $M$ has only a finite number of direct summands which are nonzero.

If $M$ is a right $R$-module and $x_{1}, \ldots, x_{n} \in M$, then $\left[x_{1}, \ldots, x_{n}\right]$ will denote the submodule of $M$ generated by $\left\{x_{1}, \ldots, x_{n}\right\}$. For $x \in M, x R=\{x r \mid r \in R\}$. If $R$ is a ring with identity and $M$ a unitary $R$-module, then clearly $[x]=x R$. A module $M_{R}$ will be called regular if for $0 \neq x \in M, x R \neq 0$. Clearly if $R$ is a ring with identity every unitary $R$-module is regular. Also if $R$ is arbitrary then $M_{R}$ is regular if $Z\left(M_{R}\right)=0$.

THEOREM 1.3. Let $M_{R}$ be a right $R$-module, and consider the following conditions.

(a) $\operatorname{dim} M_{R}$ is finite.

(b) If $K \in L\left(M_{R}\right)$, there are $x_{1}, \ldots, x_{n} \in K$ such that $\left[x_{1}, \ldots, x_{n}\right] \in L\left(M_{R}\right)$.

(c) If $K$ is a submodule of $M$, then there are $x_{1}, \ldots, x_{n} \in K$ such that $\left[x_{1}, \ldots, x_{n}\right]$ $\in L\left(K_{R}\right)$

(b*) If $K \in L\left(M_{R}\right)$, there are $x_{1}, \ldots, x_{n} \in K$ such that $\sum x_{i} R \in L\left(M_{R}\right)$.

(c*) If $K$ is a submodule of $M$, then there are $x_{1}, \ldots, x_{n} \in K$ such that $\sum x_{i} R$ $\in L\left(K_{R}\right)$.

If $M_{R}$ is any $R$-module then (a), (b), and (c) are equivalent. If $M_{R}$ is a regular $R$-module then (a), ( $\left.\mathrm{b}^{*}\right)$, and $\left(\mathrm{c}^{*}\right)$ are equivalent, hence all the statements are equivalent.

Proof. Only the equivalence of (a), (b), and (c) will be shown as the equivalence of (a), ( $\left.\mathrm{b}^{*}\right)$, and ( $\left.\mathrm{c}^{*}\right)$ when $M_{R}$ is regular has an analogous proof.

(a) implies (b). Let $K \in L\left(M_{R}\right)$ and suppose (b) is denied. Let $0 \neq x_{1} \in K$, then $\left[x_{1}\right] \notin L\left(K_{R}\right)$, hence there is $0 \neq x_{2} \in K$ such that $\left[x_{1}\right] \cap\left[x_{2}\right]=0$. Suppose $x_{1}, \ldots, x_{n}$ $\in K$ such that $0 \neq\left[x_{i}\right]$ and the sum $\sum\left[x_{i}\right]$ is direct. Since $\sum\left[x_{i}\right]=\left[x_{1}, \ldots, x_{n}\right]$, $\sum\left[x_{i}\right] \notin L\left(K_{R}\right)$, hence there is $0 \neq x_{n+1} \in K$ such that $\left[x_{1}, \ldots, x_{n}\right] \cap\left[x_{n+1}\right]=0$. Thus, there is an infinite sum $\left[x_{1}\right] \oplus \cdots \oplus\left[x_{n}\right] \oplus \cdots \subseteq K \subseteq M$ contradicting (a), so for some $n\left[x_{1}, \ldots, x_{n}\right] \in L\left(K_{R}\right)$, hence $\left[x_{1}, \ldots, x_{n}\right] \in L\left(M_{R}\right)$ since $K \in L\left(M_{R}\right)$.

(b) implies (c). Let $K$ be a submodule of $M_{R}$. By Zorn's lemma there is a submodule $L$ of $M$ which is maximal with respect to the property that $K \cap L=0$ and consequently $K+L \in L\left(M_{R}\right)$. By (b) there exist finitely many $a_{1}, \ldots, a_{n} \in K+L$ such that $\left[a_{1}, \ldots, a_{n}\right] \in L\left(M_{R}\right)$. Now $a_{i}=x_{i}+y_{i}, k_{i} \in K, y_{i} \in L$. The counter image of $\left[x_{i}, y_{i}\right]_{i=1}^{n} \in L\left(M_{R}\right)$ by the inclusion map $K \rightarrow K+L$ is $\left[x_{1}, \ldots, x_{n}\right]$, hence is large in $K$, so (c) follows. 
(c) implies (a). Given $K$ a direct sum of nonzero submodules of $M$, then there are finitely many $x_{1}, \ldots, x_{n} \in K$ such that $\left[x_{1}, \ldots, x_{n}\right] \in L\left(K_{R}\right)$. Now $\left[x_{1}, \ldots, x_{n}\right]$ is contained in the direct sum of finitely many of the submodules of $K$ and $\left[x_{1}, \ldots, x_{n}\right]$ thus has zero intersection with the others so the others are zero and (a) follows.

Similar proofs show the equivalence of $(a),\left(b^{*}\right)$, and $\left(c^{*}\right)$.

Proposition 1.4. For a unitary module $A_{R}$, the following statements are equivalent.

(a) $A$ is semisimple (sum of simple submodules).

(b) $A$ is a direct sum of simple submodules.

(c) Every submodule of $A_{R}$ is a direct summand of $A$.

(d) $L\left(A_{R}\right)=\left\{A_{R}\right\}$.

Proof. The equivalence of (a), (b), and (c) is well known, e.g., [8]. Clearly (c) implies (d). Conversely, if $B$ is a submodule of $A$, then there is a submodule $C$ of $A$ such that $B \cap C=0$ and $B+C \in L\left(A_{R}\right)$, by (d) $A=B \oplus C$ so (c) follows.

LEMMA 1.5. If $Q$ is a right quotient ring of $R$ with $Z\left(R_{R}\right)=0$ and $A, B R$-submodules of $Q_{R}$ such that $A \cap B=0$, then $A Q \cap B Q=0$.

Proof. If $x \in A Q \cap B Q$, then $x=\sum a_{i} q_{i}=\sum b_{i} p_{i}, a_{1}, \ldots, a_{n} \in A, b_{1}, \ldots, b_{n} \in B$, $p_{1}, \ldots, p_{n}, q_{1}, \ldots, q_{n} \in Q . I=\left\{r \in R \mid q_{i} r \in R, p_{i} r \in R\right.$ for all $\left.i\right\} \in L\left(R_{R}\right)$ and $x I=0$ so $x \in Z\left(Q_{R}\right)=0$, since $Z\left(R_{R}\right)=0$.

COROLlaRY. If $Q$ is a right quotient ring of $R, Z\left(R_{R}\right)=0$ and $B \in L\left(Q_{Q}\right)$, then $B \cap L\left(R_{R}\right)$.

Proof. If $B \cap J=0$ for a right ideal $J$ of $R$, then $B Q \cap J Q=0$ by the lemma, hence $J=0$ since $B Q=B$.

THEOREM 1.6. Let $Z\left(R_{R}\right)=0$, and $Q$ the maximal right quotient ring of $R$, then the following statements are equivalent.

(a) $I Q=Q$ for every $I \in L\left(R_{R}\right)$.

(b) For $I \in L\left(R_{R}\right)$ there are $a_{1}, \ldots, a_{n} \in I$ such that $\sum a_{i} R \in L\left(R_{R}\right)$.

(c) $\operatorname{dim} R_{R}$ is finite.

(d) If $I$ is a right ideal of $R$, then there are $a_{1}, \ldots, a_{n} \in I$ such that $\sum a_{i} R \in L\left(I_{R}\right)$.

(e) $Q$ is a semisimple ring.

Proof. The equivalence of (b), (c), and (d) follows from Theorem 1.3 since $Z\left(R_{R}\right)=0$.

(a) implies (b). If $I \in L\left(R_{R}\right)$, then $I Q=Q$, hence there are $a_{1}, \ldots, a_{n} \in I, q_{1}, \ldots, q_{n}$ $\in Q$ such that $\sum a_{i} q_{i}=1 . J=\left\{r \in R \mid q_{i} r \in R\right.$ for all $\left.i\right\} \in L\left(R_{R}\right)$ and clearly $J \subseteq \sum a_{i} R$ so (b) follows.

(b) implies (e). If $B \in L\left(Q_{Q}\right)$, then $B \cap R \in L\left(R_{R}\right)$ by the corollary to Lemma 1.5. So $B \cap R$ has elements $a_{1}, \ldots, a_{n}$ such that $I=\sum a_{i} R \in L\left(R_{R}\right) . I Q$ is a finitely generated right ideal of $Q$. Since $Z\left(R_{R}\right)=0, Q$ is a regular ring, hence $I Q=e Q$, $e=e^{2} \in Q$. However, $(1-e) I=0$ so $1-e \in Z\left(Q_{R}\right)=0$ so $I Q=Q$, but $I Q \subseteq B$ so $B=Q$, that is $L\left(Q_{Q}\right)=\left\{Q_{Q}\right\}$ so $Q$ is a semisimple ring. 
(e) implies (a). If $I \in L\left(R_{R}\right)$, then $I Q=e Q$ for some $e=e^{2} \in Q$ since $Q$ is semisimple. Since $Q$ is the maximal right quotient ring of $R$ and $Q$ is semisimple, then $Q$ is a regular ring so $Z\left(Q_{R}\right)=0$ by [9]. Therefore, since $(1-e) I=0,(1-e) \in Z\left(Q_{R}\right)$ $=0$, so $1=e$ and $I Q=Q$.

Now the case of a semisimple classical right quotient ring of $R$ will be considered.

THEOREM 1.7. For a ring $R$, the following statements are equivalent.

(a) $R$ has a semisimple classical right quotient ring.

(b) $Z\left(R_{R}\right)=0$ and for $I \in L\left(R_{R}\right)$ there is a $\in I$ such that $a R \in L\left(R_{R}\right)$.

(c) $R$ is a semiprime ring, $\operatorname{dim} R_{R}$ is finite and $R$ satisfies the ascending chain condition on right annihilators.

Proof. The equivalence of (a) and (c) was shown by Goldie [7].

(a) implies (b) $\left({ }^{2}\right)$. Let $Q$ be a semisimple classical right quotient ring of $R$, then $Q$ is the maximal right quotient ring of $R$. By Theorem $1.6 Z\left(R_{R}\right)=0$ and for $I \in L\left(R_{R}\right)$, there are $a_{1}, \ldots, a_{n} \in I, q_{1}, \ldots, q_{n} \in Q$ such that $\sum a_{i} q_{i}=1$. Since $q_{1} \in Q$, $q_{1}=c_{1} d_{1}^{-1}, c_{1}, d_{1} \in R, d_{1}$ regular in $R$, hence $d_{1}=\sum_{i>1} a_{i} q_{i} d_{1}+a_{1} c_{1}$. Since $q_{2} d_{1} \in Q$, $q_{2} d_{1}=c_{2} d_{2}^{-1}, c_{2}, d_{2} \in R, d_{2}$ regular in $R$, so $d_{1} d_{2}=a_{1} c_{1} d_{2}+a_{2} c_{2}+\sum_{i>2} a_{i} q_{i} d_{1} d_{2}$. Continuing in this fashion it follows that there exist regular elements $d_{1}, \ldots, d_{n} \in R$ such that $d=d_{1} \cdots d_{n} \in \sum a_{\mathfrak{i}} R \subseteq I$. If $d R \cap J=0$ for a right ideal $J$ of $R$, then $d R Q \cap J Q=0$, but since $R \in L\left(R_{R}\right), R Q=Q$ so $d R Q=Q$ since $d$ is regular so $J=0$, hence $d R \in L\left(R_{R}\right)$.

(b) implies (a). Let $Q$ be the maximal right quotient ring of $R$ and $q \in Q$, then $I=\{r \in R \mid q r \in R\} \in L\left(R_{R}\right)$. By (b) there is $a \in I$ such that $a R \in L\left(R_{R}\right)$. By Theorem 1.6 $Q=a R Q=a Q$ so $a$ has right inverse. Since $Q$ is semisimple and $a$ has a right inverse $a$ has a left inverse so $a$ is a regular element of $R$ and $q=b a^{-1}$. If $a \in R$ and $a$ is regular, then the right annihilator of $a$ in $R$ is zero, hence in $Q$ also. Since $Q$ is semisimple $a$ is regular in $Q$ and (a) follows.

It is not valid in general that for a right $R$-module $A_{R}, Z\left(A / Z\left(A_{R}\right)\right)=0$. Let $R$ be a local ring with Jacobson radical $N \neq 0$ such that $N^{2}=0$. For instance $I /\left(p^{2}\right), \dot{I}$ the ring of integers and $p$ a prime. Since $N$ is the unique maximal right ideal of $R$, $N \in L\left(R_{R}\right)$, hence it follows that $Z\left(R_{R}\right)=N$. Since $(R / N) N=0, Z(R / N)=R / N \neq 0$.

THEOREM 1.8. If $Z\left(R_{R}\right)=0$ and $\operatorname{dim} R_{R}$ is finite, then $Z(A / Z(A))=0$ for every right $R$-module $A_{R}$.

Proof. If $x+Z(A) \in Z(A \mid Z(A))$, then $I=\{r \in R \mid x r \in Z(A)\} \in L\left(R_{R}\right)$ by definition. By Theorem 1.6, $Q$, the maximal right quotient ring of $R$ is semisimple so $I Q=Q$, hence there are $a_{1}, \ldots, a_{n} \in I, q_{1}, \ldots, q_{n} \in Q$ such that $\sum a_{i} q_{i}=1$. For each $i$, $x a_{i} \in Z(A)$ so $I_{i}$, the annihilator of $x a_{i}$ in $R$, is in $L\left(R_{R}\right)$. By the corollary to Proposition $1.2 J=\left\{r \in R \mid q_{i} r \in I_{i}\right.$ for each $\left.i\right\} \in L\left(R_{R}\right)$. For $r \in J, x r=x\left(\sum a_{i}\left(q_{i} r\right)\right)=\sum x a_{i}\left(q_{i} r\right)$ $=0$, so $x \in Z(A)$ and the theorem follows.

$\left.{ }^{2}\right)$ Goldie [7] has shown this implication also. 
This theorem raises the question of whether or not the condition $Z(A / Z(A))=0$ for every $R$-module is sufficient for $R$ to possess a semisimple maximal ring of quotients.

2. Rings with identity. In this section $R$ is a ring with unity 1 , and all right $R$-modules are unitary.

LeMma 2.1. Let $Q$ be a right quotient ring of $R$ and $A$ a right $R$-module, then if $a \otimes 1=0$ in $A \otimes_{R} Q$, there are finitely many $q_{i} \in Q, a_{j} \in A, a=a_{1},\left\{r_{i j}\right\} \subseteq R$ such that

$$
\sum_{i} r_{i j} q_{i}=\delta_{1 j} \quad(\text { Kronecker delta })
$$

and $\sum_{j} a_{j} r_{i j}=0$ for all $i$.

Proof. Let $F$ be a free right $R$-module with basis $\left\{x_{a}: a \in A\right\}$, then the sequence

$$
0 \rightarrow K \rightarrow F \rightarrow A \rightarrow 0
$$

of right $R$-modules is exact, where $(F \rightarrow A)\left(x_{a}\right)=a, K=\operatorname{Ker}(F \rightarrow A)$. Tensoring over $R$ with $Q$ we have the exact sequence

$$
K \otimes Q \rightarrow F \otimes Q \rightarrow A \otimes Q \rightarrow 0 .
$$

If $a \otimes 1=0$ in $A \otimes Q$, then $x_{a} \otimes 1 i \mathrm{~s}$ the image of an element from $K \otimes Q$. $x_{a} \otimes 1=\sum_{i} k_{i} \otimes q_{i}$. Since $k_{i} \in K \subseteq F, k_{i}=\sum_{j} x_{a j} \lambda_{i j}$ for each $i$, a finite sum. Now $x_{a} \otimes 1=\sum_{i}\left(\sum_{j} x_{a j} \lambda_{i j}\right) \otimes q_{i}=\sum_{j} x_{a j} \otimes\left(\sum_{i} \lambda_{i j} q_{i}\right)$. Since representation in $F \otimes Q$ is unique with respect to basis elements $x_{a}=x_{a j}$ for some $j$ say $j=1$, hence $\sum_{i} \lambda_{i j} q_{i}=\delta_{1_{j}}$ and from $k_{i}=\sum_{j} x_{a_{j}} \lambda_{i j}, 0=\sum_{j} a_{j} \lambda_{i j}$ for all $i$, and the lemma follows.

Proposition 2.2. If $Q$ is a right ring of quotients of $R, A$ a right $R$-module, then the kernel of the map $A \otimes R \rightarrow A \otimes Q$ is contained in $Z\left(A_{R}\right)$.

Proof. If $a \otimes 1=0$ in $A \otimes Q$, then by Lemma 1.3 there exist finitely many $\left\{q_{i}\right\} \subseteq Q,\left\{a_{j}\right\} \subseteq A, a_{1}=a,\left\{\lambda_{i j}\right\} \subseteq R$ such that

$$
\sum_{i} \lambda_{i j} q_{i}=\delta_{1 j}, \quad \sum_{j} a_{j} \lambda_{i j}=0 \text { for all } i .
$$

Let $I=\left\{r \in R \mid q_{i} r \in R\right.$ for each $\left.i\right\}$, then $I$ is a large right ideal of $R$ by the corollary to Proposition 1.2 and for $\lambda \in I$

$$
\begin{aligned}
0 & =\sum_{i}\left(\sum_{j} a_{j} \lambda_{i j}\right)\left(q_{i} \lambda\right)=\sum_{i, j} a_{j} \lambda_{i j}\left(q_{i} \lambda\right) \\
& =\sum_{j} a_{j}\left(\sum \lambda_{i j}\left(q_{i} \lambda\right)\right)=\sum_{j} a_{j}\left(\delta_{1 j} \lambda\right)=a_{1} \lambda=a \lambda,
\end{aligned}
$$

hence $a$ is annihilated by $I$, so $a \in Z\left(A_{R}\right)$.

THEOREM 2.3. If $Q$ is the maximal right quotient ring of $R$, then (a), (b), (c), (d), (e) of Theorem 1.6 are all equivalent to (f) $\operatorname{Ker}\left(A \otimes_{R} R \rightarrow A \otimes_{R} Q\right)=Z\left(A_{R}\right)$ for every right $R$-module $A$. 
Proof. (a) implies (f). By Proposition 2.2, $\operatorname{Ker}(A \otimes R \rightarrow A \otimes Q) \subseteq Z\left(A_{R}\right)$. If $a \in Z\left(A_{R}\right)$ then $I$, the annihilator of $a$ in $R$, is in $L\left(R_{R}\right)$ so $I Q=Q$, hence there are $a_{1}, \ldots, a_{n} \in I, q_{1}, \ldots, q_{n} \in Q$ such that $\sum a_{i} q_{i}=1$. In $A \otimes Q, a \otimes 1=a \otimes\left(\sum a_{i} q_{i}\right)$ $=\sum a a_{i} \otimes q_{i}=0$ so (f) follows.

(f) implies (a). If $I \in L\left(R_{R}\right)$, then from the exact sequence $I \otimes Q \rightarrow R \otimes Q$ $\rightarrow R / I \otimes Q \rightarrow 0$ we have that $R / I \otimes Q$ is isomorphic to $Q / I Q$. Since $I \in L\left(R_{R}\right)$ $Z(R / I)=R / I$, so $R / I \otimes R \rightarrow R / I \otimes Q$ is the zero map hence $\overline{1} \otimes 1=0$ in $R / I \otimes Q$. However, $R / I \otimes Q \cong Q / I Q$ is a right $Q$-module generated by $\overline{1} \otimes 1$ so $Q / I Q=0$, hence $I \otimes Q \rightarrow Q$ is onto. It is now clear that (a) follows since the image of $I \otimes Q \rightarrow Q$ in $Q$ is $I Q$.

An immediate consequence of the notion of singular submodule is

Proposition 2.4. If $E_{R}=\oplus_{I} E_{i}, E_{i}$ right $R$-modules then $Z(E)=\oplus_{I} Z\left(E_{i}\right)$.

If $R$ is a commutative integral domain, then any direct sum of torsion free injective $R$-modules is injective, since it is torsion-free and divisible, hence injective, [1, Proposition VII.1.3]. A generalization holds.

THEOREM 2.5. If $\operatorname{dim} R_{R}$ is finite and $E_{R}$ is the direct sum of injectives which have zero singular submodule, then $E$ is injective.

Proof. By Proposition 2.3 $Z(E)=0$. It is sufficient to show that every $R$-homomorphism from a large right ideal of $R$ into $E$ can be extended to $R$. Let $f$ $\in \operatorname{Hom}\left(I_{R}, E_{R}\right), I_{R} \in L\left(R_{R}\right)$. By Theorem 1.3, there exist finitely many $a_{1}, \ldots, a_{n} \in I$ such that $J=\sum a_{i} R \in L\left(R_{R}\right)$. Let $f^{\prime}$ be the restriction of $f$ to $J$. Since $J$ is finitely generated, $f^{\prime}(J)$ is contained in a finite direct sum of injectives, hence $f^{\prime}$ has an extension $f^{*} \in \operatorname{Hom}\left(R_{R}, E_{R}\right)$. The assertion is that $f^{*}$ is an extension of $f$. Let $x \in I$, then $K=\{r \in R \mid x r \in J\} \in L\left(R_{R}\right)$. Now for $r \in K,\left(f(x)-f^{*}(x)\right) r=f(x r)-f^{*}(x r)$ $=f^{\prime}(x r)-f^{\prime}(x r)=0$ so $f(x)-f^{*}(x) \in Z(E)=0$, hence $f^{*}(x)=f(x)$.

It is known [3], that if $Z\left(R_{R}\right)=0$, then $Q$ the maximal right ring of $R$ is injective as a right $R$-module.

THEOREM 2.6. If $Q$ is the maximal right quotient ring of $R, Z\left(R_{R}\right)=0, \operatorname{dim} R_{R}$ finite and $A_{R}$ a right $R$-module such that $Z\left(A_{R}\right)=0$, then the map $0 \rightarrow A \rightarrow A \otimes_{R} Q$ is an injective hull of $A$ as a right $R$-module.

Proof. The map is a monomorphism by Theorem 2.3 .

Now $A \otimes_{R} Q$ is a right $Q$ module, hence semisimple since $Q$ is, so $A \otimes_{R} Q$ is a direct sum of direct summands of $Q$. Since $Z\left(Q_{R}\right)=0, Z\left(A \otimes_{R} Q\right)=0$ regarding $A \otimes Q$ as a right $R$-module and by Theorem $2.5 A \otimes_{R} Q$ is injective as a right $R$-module.

If $0 \neq x=\sum a_{i} \otimes q_{i} \in A \otimes Q$, then $I=\left\{r \in R \mid q_{i} r \in R\right\} \in L\left(R_{R}\right)$. Now $0 \neq x I$ since $Z(A \otimes Q)=0$ and $x I \subseteq \operatorname{Im}(A \rightarrow A \otimes Q)$ so $0 \rightarrow A \rightarrow A \otimes Q$ is an essential monomorphism, i.e., $\operatorname{Im}(A \rightarrow A \otimes Q)$ is a large right $R$ submodule of $A \otimes Q$ and the theorem follows. 
THEOREM 2.7. If $Q$ is a semisimple maximal right quotient ring of $R$, then $Q$ is flat as a left $R$-module.

Proof. It is sufficient to show that $I \otimes_{R} Q \rightarrow R \otimes_{R} Q$ is a monomorphism for every right ideal of $R$. As before $I \otimes_{R} Q$ is a right $Q$-module, then $Z(I \otimes Q)=0$ regarding $I \otimes Q$ as a right $R$-module. If $x=\sum a_{i} \otimes q_{i} \in \operatorname{Ker}(I \otimes Q \rightarrow R \otimes Q)$, then $I=\left\{r \in R \mid q_{i} r \in R\right\} \in L\left(R_{R}\right)$ and $\sum a_{i} q_{i}=0$ in $Q$. Clearly $x I=0$, so $x \in Z(I \otimes Q)=0$, so $x=0$, hence $I \otimes Q \rightarrow R \otimes Q$ is a monomorphism.

COROLlaRY. If $Q$ is the maximal right quotient ring of $R, Z\left(R_{R}\right)=0, \operatorname{dim} R_{R}$ finite, then $\operatorname{Tor}_{1}^{R}(A, Q / R) \cong Z\left(A_{R}\right)$ for every right $R$-module $A_{R}$.

Proof. It follows from the exact sequence $\operatorname{Tor}_{1}^{R}(A, Q) \rightarrow \operatorname{Tor}_{1}^{R}(A, Q / R) \rightarrow A$ $\otimes_{R} R \rightarrow A \otimes_{R} Q$ since by the theorem $\operatorname{Tor}_{1}^{R}(A, Q)=0$ and $\operatorname{Ker}\left(A \otimes_{R} R \rightarrow A \otimes_{R} Q\right)$ $\cong Z\left(A_{R}\right)$ by Theorem 2.3 .

COROLlaRY. If $Q$ is a semisimple maximal right quotient of $R$, then every left $Q$-module is flat as a left $R$-module.

Proof. Every left $Q$-module is a direct sum of direct summands of $Q$, hence is flat as a left $R$-module since $\operatorname{Tor}_{n}^{R}$ commutes with direct sum $s$.

Matlis [12, Theorem 1.1] has shown that if $R$ is a commutative integral domain and $H$ an $R$-module, then the torsion submodule of $H$ is a direct summand of $H$, if $H$ is an epimorphic image of an injective $R$-module. This result is generalized and the proof does not appeal to the quotient ring of $R$.

First, the notion of a closed submodule of a module will be considered and some consequences. Johnson and Wong [11] considered the notion of a closed submodule.

Definition 2.8. A submodule $B$ of a module $A$ is closed if $B$ has no essential extension in $A$; i.e., $C$ a submodule of $A$ such that $B$ is a large submodule of $C$ implies $B=C$.

Remark. If $E$ is an injective $R$-module and $A$ a submodule of $E$, then $A$ is closed if and only if $A$ is a direct summand of $E$. This follows from the fact that every submodule of $E$ is a large submodule of its injective hull in $E$, which is a direct summand of $E$.

Lemma 2.9. If $f \in \operatorname{Hom}(M, A), B$ a submodule of $A$ such that $Z(A \mid B)=0$, then $f^{-1}(B)$ is a closed submodule of $M$.

Proof. Let $D$ be a submodule of $M$ containing $f^{-1}(B)$ as a large submodule. If $d \in D$, then $I=\left\{r \in R \mid d r \in f^{-1}(B)\right\} \in L\left(R_{R}\right)$. Now $f(d) I=f(d I) \subseteq B$, hence $f(d)$ $+B=[f(d)]^{-} \in Z(A / B)=0$ so $f(d) \in B$ and $d \in f^{-1}(B)$ and the lemma follows.

THEOREM 2.10. If $E \rightarrow^{f} H \rightarrow 0$ is an exact sequence of right $R$-module, $E$ injective, $Z(H / Z(H))=0$, then $H=Z(H) \oplus F$ and $F$ is injective.

Proof. Since $Z(H / Z(H))=0$, by Lemma $2.9 f^{-1}(Z(H))$ is closed in $E$, hence a direct summand of $E=f^{-1}(Z(H)) \oplus G$. Clearly $H=Z(H) \oplus f(G)$ and $f(G) \cong G$. 
COROllaRY. If $R$ is a ring such that $Z\left(R_{R}\right)=0, \operatorname{dim} R_{R}$ finite, then every epimorphic image of an injective $R$-module has its singular submodule as a direct summand.

Proof. An immediate consequence of the theorem and Theorem 1.8.

It is interesting to note that some of the propositions of [12] admit generalizations to noncommutative rings and their maximal right quotient rings, where torsion submodule is replaced with singular submodule and $Q$ the maximal right quotient ring of $R$.

If in addition to the hypothesis of [12, Proposition 2.1] we assume $Z\left(R_{R}\right)=0$, $\operatorname{dim} R_{R}$ finite, then Proposition 2.1 is valid with the same proofs using the fact that $Z\left(Q_{R}\right)=0$ and a direct sum of copies of $Q$ is injective by Theorem 2.5. Similarly a generalization of [12, Proposition 2.2] is valid in view of the corollary to Theorem 2.11, as well as [12, Corollary 2.3] of [12, Proposition 2.2].

\section{BIBLIOGRAPHY}

1. H. Cartan and S. Eilenberg, Homological algebra, Princeton Univ. Press, Princeton, N. J., 1956.

2. B. Eckmann and A. Schopf, Uber injektive Modulen, Arch. Math. 4 (1953), 75-78.

3. C. Faith, Injective modules and quotient rings, Lecture Notes, Rutgers, The State University, New Brunswick, N. J., 1964.

4. G. D. Findlay and J. Lambek, A generalized ring of quotients. I, II, Canad. Math. Bull. 2 (1958), 77-85, 155-167.

5. E. Gentile, On rings with one-sided field of quotients, Proc. Amer. Math. Soc. 11 (1960), 380-384.

6. A. W. Goldie, The structure of prime rings under ascending chain conditions, Proc. Lond. Math. Soc. 8 (1958), 589-608.

7. - Semi-prime rings with maximum condition, Proc. London Math. Soc. 10 (1960), 201-220.

8. N. Jacobson, Structure of rings, Colloq. Publ., Vol. 37, Amer. Math. Soc., Providence, R. I., 1956.

9. R. E. Johnson, The extended centralizer of a ring over a module, Proc. Amer. Math. Soc. 2 (1951), 891-895.

10. R. E. Johnson and E. T. Wong, Self-injective rings, Canad. Math. Bull. 2 (1959), 167-173.

11. _ Quasi-injective modules and irreducible rings, J. London Math. Soc. 36 (1961), 260-268.

12. E. Matlis, Divisible modules, Proc. Amer. Math. Soc. 11 (1960), 385-391.

13. J. von Neumann, On regular rings, Proc. Nat. Acad. Sci. U. S. A. 22 (1936), 707-713.

14. Y. Utumi, On quotient rings, Osaka Math. J. 8 (1956), 1-18.

UNIVERSITY OF WISCONSIN, Madison, Wisconsin 